\title{
Biochar as a cheap and environmental friendly filler able to improve T polymer mechanical properties
}

\author{
Mauro Giorcellia, Aamer Khana, Nicola M. Pugno, Carlo Rosso, Alberto Tagliaferro
}

\section{Abstract}

This study reports about the use of Biochar derived from maple tree as a filler in Epoxy resin. Maple tree blocks were pyrolyzed in inert atmosphere at $600{ }^{\circ} \mathrm{C}$ and $1000{ }^{\circ} \mathrm{C}$ respectively and were characterized morphologically. The composite mechanical properties, i.e. stress-strain curves and related parameters (ultimate tensile strength, Young modulus, resilience, tensile toughness) were recorded as well as their friction coefficient. It is shown that at very low wt.\% of the filler, the Young modulus is increased while at higher wt.\% ( $2 \mathrm{wt} \%$ and above) the fragile behavior of the resin was converted in a ductile one, as elongation at break increased from 0.02 to 0.12 . A huge impact of the filler is observed on tensile toughness as for the best sample is increased 11 times with respect with pure resin. A simple model able to describe the results and make predictions on other wt.\% is presented as well.

\section{Introduction}

From the last several years, biomass is increasingly recognized as a valuable commodity. In particular, lignocellulosic biomass, the most abundant organic materials on earth, has enormous potential as a feedstock for the production of fuel, heat and electrical power. In ser- ious consideration of the worldwide economic and environmental pol- lution issues there has been increasing research interest in the value all the product of this conversion (liquid, gas, solid). In particular, solid residual of lignocellulosic biomasses conversion is called Biochar. This by-product found its main application as soil remediation but recently alternative applications were proposed. Nanda et al. [1] summarize results of Biochar application in different fields as Energy, Agronomy, Carbon Sequestration, Activated Carbon and Speciality Materials as composites. As Biochar is rich in carbon, several studies try to explore and compare its properties with those of more expensive carbon ma- terial, for instance in electronic application [2]. It was also demonstrated the possibility to apply Biochar in fields such as sensors where only traditional carbon materials were applied [3,4]. In other fields such as constructions Biochar was considered [5] also because of its low cost, since the economical aspect is critical. More in general Biochar is finding applications in composite/polymer sector [6]. In this field, Biochar was able to increase mechanical, electrical and thermal properties [7].

As mentioned studies reported, the possible applications of this low cost and eco-friendly carbon based material are in different fields and Biochar could became a leader among carbon materials in composites. 
In this article, we report a morphological characterization of Biochar and Biochar heat treated (HT) in order to evaluate differences. Biochars were then dispersed in an epoxy resin to produce composites. For composites we report a complete investigation on the mechanical point of view (ultimate tensile strength, Young modulus, resilience, tensile toughness) comparing the influence of these two types of Biochar. Finally, a calculation of the efficiency of the different Biochar content was done.

\section{Materials and methods}

Two types of Biochar, both derived from maple tree, were provided by University of Toronto. The two types of Biochar, labelled Biochar and Biochar $\mathrm{HT}$ in the following were pyrolyzed in inert atmosphere at $600^{\circ} \mathrm{C}$ and $1000^{\circ} \mathrm{C}$ respectively. Biochar and Biochar HT were grinded using a mechanical mixer (Savatec BB90E) in order to obtain a fine powder (average particle size $10 \mu \mathrm{m}$ ) easy to uniformly disperse in composite. Low viscosity Epoxy resin LPL (Cores Ocean) was used to produce composites in dog bone shape for mechanical tests. Morphology of Biochar, Biochar HT and composites was investigated by Field Emission Scanning Electron Microscope (FESEM-ZEISS SUPRA- 40TM). In case of composites a thin layer of few nm of chromium was used to avoid charging effects during FESEM measurements.

\subsection{Composite preparation}

Biochar composites based on epoxy resin were prepared according to the procedure detailed in Ref. [2]. Briefly, the appropriate quantity of Biochar powder as reported in Table 1 was dispersed in low viscosity epoxy resin using ULTRATURRAX (T18) at 20,000 rpm for $2 \mathrm{~min}$. Hardener was then added and the final composites mixed using a me- chanical low speed mixer in order to obtain a uniform dispersion of the components. Finally, the liquid composite was sonicated (Elma sonic $\mathrm{S} 15 \mathrm{H}$ ) for $5 \mathrm{~min}$ and then degassed in a vacuum chamber ( $50 \mathrm{mbar}$ ) for 10 further minutes to remove gas bubbles created during the mixing process. The composite mixture was molded in dogbone shape and cured in oven at $60{ }^{\circ} \mathrm{C}$ for $4 \mathrm{~h}$. Five samples for each concentration were prepared to test reproducibility.

\subsection{Composite analysis}

The tensile behavior of the composites was studied using a tensile testing machine (MTS Q-test10). All specimens were measured with a load cell of $10 \mathrm{kN}$ and a strain rate of $5 \mathrm{~mm} / \mathrm{min}$ according to ASTM D- 638-4 standard. Specimen broken in the central region were considered for further analysis. Stress vs strain data were recorded and compared with blank epoxy resin values.

\section{Results and discussions}

\subsection{Morphology of Biochar and Biochar HT}

Small quantities of Biochar and Biochar HT in powder form were positioned on the sample holder using a conductive tape. The morphology of Biochar was then studied using FESEM. Biochar and Biochar HT were analyzed prior to mechanical grinding in order to study the structure of the material. In Fig. 1 we report four significant images. Lower magnification images show a similar structure for the two Bio- chars: smooth lateral surfaces and long channels. At high magnification we observe that the diameters of the channels increase by a few mi-crometers after high temperature treatment.

\subsection{Morphology of the composites after tensile testing}

Dispersion of the Biochar and Biochar HT particles in the epoxy matrix is depicted in Fig. 2. Biochar and Biochar HT particles are dis- persed evenly and uniformly throughout the matrix. Large agglomera- tions can be however identified 
in the specimen containing higher percentages of filler due to their high volume and surface area (Fig. 2eand f). However, the original porous morphology of the Biochar and Biochar HT and channels are lost after grinding to fine powder and does not seem to change the composite mechanical properties sig- nificantly as evident in the stress-strain diagrams (Fig. $3)$. The Biochar particles are well anchored in the matrix, hindering the crack propa- gation and accumulation as shown in the FESEM images. This effect can promote good surface mechanical interlocking that leads to better tensile properties.

\subsection{Mechanical analysis}

Stress-strain tensile behaviours for 1, 2, 3, 4, $20 \mathrm{wt} \%$ Biochar and Biochar HT samples are depicted in Fig. 3. The curve of pure Epoxy resin is reported as well for comparison. Addition of $1 \mathrm{wt} \%$ of Biochar filler did not alter the brittleness of the matrix. A slight increase in the strain at break along with a $63 \%$ increase in ultimate tensile strength was recorded for Biochar $1 \mathrm{wt} \%$ specimen. Addition of higher Biochar amounts (2-4 wt \%) modifies the behavior of composites from brittle to ductile. An increase in tensile strength and elongation of $\sim 48 \%$ and $\sim 500 \%$ respectively was observed for Biochar $2 \mathrm{wt} \%$ and Biochar HT $2 \mathrm{wt} \%$. Higher Biochar and Biochar HT contents further modified the mechanical behavior of the composites leading to reduced strength and elongation i.e. towards semi-brittle behavior. This behavior is most evident for high specific volume of the fillers (20 wt\%). Das et al., 2016 [8] reported the improved mechanical properties of the Biochar poly- propylene composites and the same semi brittle trend at high wt.\% we observed for the Biochar filler.

Comparison of the most relevant mechanical properties is discussed below.

\subsection{Ultimate tensile strength (UTS)}

Addition of the carbon fillers increased the load bearing capacity of the epoxy matrix. The best results were achieved by 1 $w t \%$ addition of either Biochar concentration. An increase of $63 \%$ in the maximum load bearing capacity has been observed. UTS values for all samples are shown in Fig. 4. The increase in the mechanical strength of the com- posite can be attributed to the fact that Biochar particles drained part of the stress from the polymeric matrix because of their higher Young's modulus and obstructed the crack onset and accumulation as evidenced by FESEM images. Cavitation/debonding of the filler from the matrix due to applied stress is also evident in the FESEM analysis (see red arrows in Fig. 4b and d). This pull out and cavitation phenomenon of the Biochar filler from the matrix during the applied stress is re-sponsible for enhanced mechanical properties $[9,10]$. The cross-link ratio of the epoxy resin may also have been enhanced by the addition of Biochar fillers which effectively block the molecular motion in the polymer matrix reducing its deformability and thus strengthening the polymer matrix [11,12].

\subsection{Young Modulus}

Addition of small amounts of Biochar enhanced the stiffness of the matrix. Addition of $1 \mathrm{wt} \%$ Biochar and Biochar HT enhanced Young modulus by $63 \%$ and $50 \%$ respectively. Higher filler contents led to lower enhancement of the stiffness. $2 \mathrm{wt} \%$ of Biochar increased the blank epoxy stiffness by $33 \%$ while $20 \mathrm{wt} \%$ Biochars loading enhanced stiffness by $41 \%$. In all cases, the addition of filler enhanced the stiffness of the material as shown in Fig. 5. The enhanced stiffness of the matrix can be attributed to stress transfer from the matrix to the Biochar filler favored by good adhesion of the resin to the Biochar and Biochar HT. Fu et al., 2008 [13] reported the same phenomena for the enhanced stiffness of a polymeric matrix by the addition of micrometer size particles. Nan et al., 2016 [14] reported a similar behavior in Biochar/ PVA composites.

\subsection{Resilience}

Resilience is the measure of the maximum energy absorbed per unit volume by a material in its elastic zone while being pulled under tensile load (i.e. the energy per unit volume it can absorb before starting plastic deformation). Addition of small amount of Biochar enhanced resilience. Biochar HT at $1 \mathrm{wt} \%$ showed the best result with a $100 \%$ increase of the resilience compared to blank epoxy. Biochar $1 \mathrm{wt} \%$ came 2 nd best enhancing resilience by $23 \%$. Other wt.\% of Biochar and Biochar HT did not affect resilience considerably. In some cases, resi- lience even decreases as showed in Fig. 6. 


\subsection{Tensile Toughness}

Tensile toughness is the measure of the maximum capacity of the material to withstand load before breakage (i.e. the energy per unit volume needed to break it). Its values for the various samples are re- ported in Fig. 7. Addition of 1 wt $\%$ led to a small enhancement of the tensile toughness. A 11-fold increase in toughness occurred on the other hand for both Biochar and Biochar when their amount was increase to $2 \mathrm{wt} \%$. For further increases of the wt.\% the load bearing capacity deteriorated. The lower tensile toughness at higher filler loading is related to the change from plastic into semibrittle behavior and the uneven scattering of large filler chunks in the matrix $[15,16]$.

\section{Discussion}

By analyzing the correlation between Biochar wt.\% and enhance- ment of the composite properties, some hints can be obtained. UTS and Young modulus variations with respect to bare resin value present a trend with respect to wt.\% quite similar. Maximum impact on the properties occur for $1 \mathrm{wt} \%$ samples while the variation in filler wt.\% becomes not too effective above $3-4 \mathrm{wt} \%$. The presence of the max- imum at $1 \mathrm{wt} \%$ suggests that two competing mechanisms are at work, the first prevailing for very low filler contents, the other for higher contents. Further investigations will be carried out to identify those mechanisms that could be related to the rheological percolation threshold as observed in nanocomposite [17].

As far as toughness and resilience are concerned, the contribution of Biochar in improving such properties can be highlighted. Toughness of the composites increases with the addition of Biochar, the maximum reached at $2 \mathrm{wt} \%$ of filler. This underlines the transformation of com- posites behavior from brittle to ductile for both fillers. The increase of toughness is mainly (although not only) due to the onset of ductile behavior as the elongation at break is largely increased. On the other hand, the maximum elongation of the elastic region is adversely af- fected and this maps into the resilience reduction since the variation of Young's modulus (apart for $1 \mathrm{wt} \%$ samples) is limited. In order to fur- ther investigate Biochars effect a comparison between resilience and UTS can be performed. It can be noticed that the two features present a specular trend, while UTS is increased the resilience is decreased. $1 \mathrm{wt} \%$ filler addition proves to be the best choice for improving elastic prop- erties, whereas higher concentrations reduce the beneficial effect of filler in term of UTS and are detrimental to resilience.

As a conclusion, it is possible to say that Biochar deeply affects the composite behavior transforming the blank epoxy from brittle to ductile composite. In order to obtain that, the needed percentage of Biochar is equal to or higher than 2 $\mathrm{wt} \%$. This percentage appears as the best compromise for obtaining an improvement in almost all mechanical features.

\subsection{Filler equivalent properties}

In Table 2 we report the calculation of the filler equivalent prop- erties $\mathrm{F}$ in the different composites of properties $\mathrm{C}$, back calculated with the well-known direct rule of mixture considering the weight fractions $\mathrm{f}$ (slightly different numbers are expected considering volume $\%$ but here we use weight fractions since exact values of densities are not available) and the matrix properties $\mathrm{M}$, i.e. $\mathrm{C}=\mathrm{Ff}+\mathrm{M}(1-\mathrm{f})$ :

$F=[C-M(1-f)] / f(1)$

Thus, negative numbers correspond to fillers seen in the composites as defects for the related property.

In bold the best performances. BCHT- 1 seems to be globally the best (i.e. 3 records, i.e. yield stress and strain as well as resilience), sur- passing also MW composites (a better comparison would require to consider even for MW the 1 and 2 wt $\%$ contents), then BC- 1 ( 2 records, i.e. strength and elastic modulus) and E-BCHT-2 (1 record, i.e. tough- ness) respectively. Note that BCHT-1 is the only ones with all positive numbers. Thus, the most efficient composite could be identified as BCHT-1. 


\section{Conclusions}

Biochar derived from Maple tree and pyrolyzed at two different temperatures, was used as filler, to prepare polymer composites based on epoxy resin. Morphological characterization on these two types of Biochar were done. Thermal treatment on Biochar permit to have two different Biochars to use in this work. Five different percentages of Biochar were used in the polymer composite and their mechanical properties were studied. In general, we observed that an addition of the carbon fillers increased the load bearing capacity of the epoxy matrix. The best results were achieved by 1 $w t \%$ addition of either Biochar concentration. Stiffness of the matrix was also enhanced by an addition of small amounts of Biochar. Addition of $1 \mathrm{wt} \%$ Biochar and Biochar HT enhanced Young modulus by $63 \%$ and $50 \%$ respectively. Higher filler contents led to lower enhancement of the stiffness. Resilience also in- crease after an addition of small amount of Biochar. In particular, Biochar HT at $1 \mathrm{wt} \%$ showed the best result with $\mathrm{n} 100 \%$ increase of the resilience compared to blank epoxy. Addition of $1 \mathrm{wt} \%$ led to a small enhancement of the tensile toughness. An increase in toughness oc- curred on the other hand for both Biochar and Biochar when their amount was increase to $2 \mathrm{wt} \%$. The mathematical model confirms ex- perimental results. As a conclusion, it is possible to say that the adding of a small percentage of Biochar modify the composite behavior from mechanical point of view. In particular, this addition affects the com- posite behavior transforming the blank epoxy from brittle to ductile composite. In order to obtain that, the needed percentage of Biochar is equal to or higher than $2 \mathrm{wt} \%$. This percentage appears as the best compromise for obtaining an improvement in almost all mechanical features.

\section{Acknowledgements}

We want to thank University of Toronto, prof. Charles Jia group for Biochar and Biochar HT supply. Salvatore Guastella and Mauro Raimando for FESEM analysis. This work was partially supported by grant 685844 (project MODCOMP) in the framework of European H2020 programme. NMP is supported by the European Commission with the Graphene Flagship Core 2 n. 785219 (WP14 "Composites") and FET Proactive "Neurofibres" n. 732344 as well as by the MIUR with the "Departments of Excellence" grant L. 232/2016 and ARS01-01384- PROSCAN.

7

[1] S. Nanda, A.K. Dalai, F. Berruti, J.A. Kozinski, Biochar as an exceptionla bioresource for energy, Agronomy, carbon sequestration, activated carbon and speciality ma- terials, Waste Biomass Valor 7 (2016) 201.

[2] M. Giorcelli, A.A. Khan, A. Tagliaferro, P. Savi, F. Berruti, Microwave character- ization of polymer composite based on Biochar: a comparison of composite beha- viour for Biochar and MWCNTs, IEEE International Nanoelectronics Conference (INEC), 2016, pp. 1-2.

[3] D. Ziegler, P. Palmero, M. Giorcelli, A. Tagliaferro, J.M. Tulliani, Biochars as in- novative humidity sensing materials, Chemosensors 5 (2017) 35.

[4] M. Noman, A. Sanginario, P. Jagdale, M. Castellino, D. Demarchi, A. Tagliaferro, Pyrolyzed bamboo electrode for electrogenerated chemiluminescence of Ru(bpy) 32+, Electrochim. Acta 133 (2014) 169173.

[5] R.A. Khushnood, A. Sajjad, L. Restuccia, C. Spoto, P. Jagadale, J.M. Tulliani, 
G.A. Ferro, Carbonized nano/microparticles for enhanced mechanical properties and electromagnetic interference shielding of cementitious materials, Front. Struct. Civ. Eng. 10 (2) (2016) 209-213.

[6] A.A. Khan, P. Jagdale, M. Rovere, M. Nogués, C. Rosso, A. Tagliaferro, Carbon from waste source: an eco-friendly way for strengthening polymer composites, Compos. B Eng. 132 (2018) 87-96.

[7] A.A. Khan, P. Savi, S. Quaranta, M. Rovere, M. Giorcelli, A. Tagliaferro, C. Rosso, C.Q. Jia, Lowcost Carbon fillers to improve mechanical properties and conductivity of polymers, Polymers 9 (12) (2017) 642.

[8] O. Das, D. Bhattacharyya, D. Hui, K.T. Lau, Mechanical and flammability char- acterizations of biochar/polypropylene bio composites, Compass Part B. 106 (2016) 120-128.

[9] A. Godara, L. Mezzo, F. Luizi, A. Warrier, S.V. Lomov, A.W. van Vuure, Influence of carbon nanotube reinforcement on the processing and the mechanical behavior of carbon fiber/epoxy composites, Carbon 47 (2009) 2914-2923, https://doi.org/10. 1016/j.carbon.2009.06.039.

[10] V. Nagarajan, A.K. Mohanty, M. Misra, Bio composites with size-fractionated bio carbon: influence of the microstructure on macroscopic properties, ACS Omega 1 (2016) 636-647.

[11] A. Allaoui, S. Baia, H.M. Cheng, J.B. Bai, Mechanical and electrical properties of a MWNT/epoxy composite, Compos. Sci. Technol. 62 (2002) 1993-1998.

[12] J.B. Bai, A. Allaoui, Effect of the length and the aggregate size of MWNTs on the improvement efficiency of the mechanical and electrical properties of nano- composites - experimental investigation, Composer Part A. 34 (2003) 689-694.

[13] S.Y. Fu, X.Q. Feng, B. Lauke, Y.W. Mai, Effects of particle size, particle/matrix in- terface adhesion and particle loading on mechanical properties of particulate- polymer composites, Composer Part B 39 (2008) 933-561.

[14] N. Nan, D.B. DeVallance, X. Xie, J. Wang, The effect of bio-carbon addition on the electrical, mechanical, and thermal properties of polyvinyl alcohol/biochar com- posites, J. Compos. Mater. 50 (9) (2016) 116-1168.

[15] J.Z. Liang, R.K.Y. Li, S.C. Tjong, Morphology and tensile properties of glass bead filled low density polyethylene composites, Polym. Test. 16 (1997) 529-548.

[16] G.M. Linda Vaisman, H. Daniel Wagner, The role of surfactants in dispersion of carbon nanotubes, Adv. Colloid Interface Sci. 128 (2006) 37-46.

[17] G.-H. Hu, P. Marchal, S. Hoppe, C. Penu, Rheology as a Tool for Studying in Situ Polymerized Carbon Nanotube Nanocomposites" 531-581 in "Rheology and Processing of Polymer Nanocomposites, Wiley and Sons, 2016. 